\title{
Buchanzeigen
}

Mankiewiez, Otto, Kunstbuch derinaen ist der gantze gründliche yolkommene rechte gewisse bericht und erweisung vnnd Lehr des Hartenn Reissenden Schmertz haftigenn Peinlichen Blasonn Steines ve $\Gamma$ fasset vnnd beschriebenn durch Georgium Bartisch Im Alten Dresden 1575. Dieser vorliegende Neudruck, der im Verlag von Oskar Goblentz 1905 erschienen ist, nimmt deshalb eine Sonderstellung unter ähnlichen neu-gefassten frühen Literaturerzeugnissen ein, weil es sich um ein Werk haodelt, das bisher nie gedruckt war, sondern nur in einem einzigen Manuskript existierte, welches Hermann Cohn in der Dresdner Bibliothek entdeckt hat. Allgemein bekannt und ein vorzüglicher Ratgeber jener Zeit war desselben Bartisch Augendienst. Was nun die Bedeutung dieses Buches betrifft, so sehe ich dieselbe weniger in derDarstellung derSteinbehandlung und demBartisch-schen Vorgehen bei dieser Krankheit, mit dem im 16. Jahrhundert so viel und oft „mit Gottes Hulfe” von reisenden Steinschneidern ausgeführten Steinschnitt, sondern ich begrüsse das Buch und Manckiewicz' fleissige Arbeit als neues willkommenes Quellenmaterial, um uns ül > er die Grösse uud Qualität des chimrgischen Könnens und der Allgemeinbildung der Bader-Chirurgen zu informieren. Dass orsteres nicht zu niedrig anzuschlagen ist, ging schon aus den Marktschreyzetteln hervor; hier sehen wir sowohl die Technik als auch das Instrumentarium eines allerdings weit über Durch-schnitt hervorragenden Schnittarztes bereits zu einer gediegenen Form ge-langt. Wir lernen aus dieser Quelle den technischen Hochstand des Chirurgen-Handwerkers des 16. Jahrhunderts kennen und können hieraus einen Rück-schluss ziehen auf die Durchschnittsleistung der deutschen Badechirurgen jener Zeit überhaupt. Es scheint sogar, dass diesem Manne eine seltene Ehrlichkeit anhaftete. Der beste Prüfstein ist sein offenes Bekenntnis der postoperatorischen Fisteln. Da sagt er: „Dieser Gebreehen und Schaden tfeget sich gar oft und viel zu.” Gleichzeitig gibt der Meister aber auch zwei ganz originelle Methoden an, wie man den Fisteln beikommen könne.

Es reiht sich dies Buch den seltenen, meist in Strassburg und Frankfurt gedruckten Schriften niederer Chirurgen an, wie des Christ of or us Wirsung Artzney Buch, 1568, Johan v. Parisiis Wundartzney 1549 u. s. w. Jedenfalls ist dieses Beispiel von rein wissenschaftlicher Gesinnung und Interesse an der Historie unseres Standee, den Mankiewiez geliefert, nachahmenswert. Vielleicht hätte er aber dem Leser die Sache dadurcli etwas erleichtern können, dass er nur ein Kapitel im Urtext gebracht und die weiteren verhochdeascht hätte. Denn das sächselnde miserabele Deutsch dieses biedern Königsbrücker ist geradezu fürchterlich.

Hollander.

Oberländer und Koilmann: Die chronische Gonorrhoe der männ-lichen Harnröhre und ihre Kom plikationen. Teil II und III Mit 98 Abbildungen und 8 Tafeln. Verlag von Georg Thieme, Leipzig 1905. Dem im Jahre 1901 erschienenen I. Teil des Werkes' ist nunmehr nach $13 / 8$ ngerer Pause der II. und III. Teil gefolgt.

Schon in der Einleitung zum I. Teil hatten die Aatoren der Anregung gedacht, welche sie dem bekannten Werke von De Keersmaecker und Buchanzeigen. 
711

Verhoogen, welches von Forchheimer ins Deutsche übersetzt worden ist, verdanken.

In der vorliegenden Form wollen die Verfasser Ärzten $\pi$ nd Studierenden gewíssermassen ein Nachschlagewerk über die Behandlung der chronischen Gonorrhoe der männlichen Harnröhre und ihrer Komplikationen nach Ober-länderschen Prinzipien vorlegen.

Gemäss dem Bestreben der Verfasser, ein Lehrbuch zu scbaffen, welches in der Hauptsache die persönlichen Ansichten der Verfasser wiederzugeben bestimmt ist, nimmt das vorliegende Werk den Vorzug einheitlicher Dar-stellung in Anspruch, will aber, wie die Verfasser ausdrücklicb betonen, auch die Verdienste, welche sich andere auf dem einschlägigen Gebiete erworben haben, rückhaltlos anerkennen. Sicberlich wird die urethroskopische TJnter-suchungsmethode, deren eminente Bedeutung für das Erkennen und die Behandlung der Harnröhrenleiden leider von einem grossen Teil der Arzte noch nicht genügend gewürdigt wird, durch die vorliegende Arbeit der um diese Spezialdisziplin so verdienten Autoren gefördert und weiter verbreitet werden. Dem obersten Prinzip jeglicher $\lambda$ 'ernunftgemässen Theorie, dass ohne ge-nügende Diagnose eine Heilung nicht möglich ist, ist durch Oberländer und seine Schüler auf dem Gebiet der Harnröhrenerkrankungen eine mächtige Förderung zuteil geworden. Nicht nur darauf kommt es an, die schädigenden Mikroorganismen und ihre Stoffwechselprodukte zu beseitigen, sondern von ebenso prinzipieller Bedeutung ist es, die durch jene gesetzten Schädigungen der betreffenden Organe festzustellen und dieselbeu durch geeignete Heil-methoden zu beseitigen. Die Feststellung dor postgonorrhoischen anatomisehen Läsionen und die Besprechnng der Methoden zu ihrer Heilung bilden den Inhalt des vorliegenden Werkes. Die näheren Details müssen in dem Buche selbst nachgelesen werden.

Das vorliegende Werk bildet unzweifelhaft eine wertvolle Bereicherung des urologischen Armamentarium medicum, wird aber auch den Praktikern eine willkommene und anregende Gelegenheit bieten, sich über den Nutzen und die-Notwendigkeit einer zielbewussten, auf wissenschaftlichen Grund-lagen ruhenden Tripperbehandlung ein richtiges Bild zu verschaffen. Das Buch ist von dem Verleger mit einer Reihe guter und instruktiver Abbildungen versehen worden, und es sind demselben die ausgezeichneten Tafeln urethroskopischer Bilder aus dem bereits genannten Werke von De Keersmaecker und Verhoogen beigefügt.

E. Frank-Berlin.

VOa ZeiSSl, Maximilian, Diagnose und Therapie des Trippers und seiner Komplikationen beim Manne und Wei be. (Urban und Schwarzenberg. Berlin und Wien 1903.)

Vorliegendes Werk bildet die zweite Auflage des von Zeissl als Separatausgabe der „Wiener Klinik” verfassten Abhandlung „Die Behandlung des männlichen Harnröhrentrippers und seiner Komplikationen". Es ist in-sofern gegen die erste Auflage bedeutend erweitert, als die Diagnose des männlichen Harnröhrentrippers und der Tripper des weiblichen Urogenitales hinzugefügt sind. Dem letzteren Teil liegt das von Dr. Felix Heymann, Berlin, für das „Lehrbuch der venerischen Krankbeiten" von Zeissl be-arbeitete Kapitel der weiblichen Gonorrhoe zugrunde. Bei der Besprechung des akuten Trippers und seiner Prophylaxe, wünscht Verfasser zur Vermeidung der Infektionsgefahr der von Frank angegebenen Methode die weiteste Verbreitnng. Hierdurch setzt er sich zu vielen Autoren in Gegensatz, welche nicht eindringlich genug vor der Anwendung der prophylaktischen Instillationen warnen können, so unter anderen Fin ger in einer kürzlich erst erschienenen Veröffentlichu $\pi$ g. Zur Behandlung empfiehlt er im akuten Stadium vor allem Spritzungen und Wasserspülungen der vorderen Harnröhre neben der Ver-

$50 *$ 


\section{B uchanzeigen.}

abreichung von Balsamicis. Ist nach 5 Wochen nur Besserung eingetreten, so geht er zu Spülungen bis in die Blase fiber zur Beseitigung der Filamente, zuletzt wendet er Antrophore und Instillationen mit starken Argentumlösungen an. Gegen die neuere $\pi$ Behandlungsmethoden, Dehnung mit Dilatatorien und die endoskopische Untersuchung verhält er sicli völlig ablelinend. Er warnt bei langdauernden, viel behandelten Trippern vor der Polypragmasie, oft er-folgt Heilung, wenn die Harnrölire in Ruhe gelassen wird. Kranke mit Fäden sind aus der Behandlung zu entlassen, wenn die häufig vorgenommene mikroskopische Untersuchung keine Gonokokken ergibt und auch Irritationen dieselben nicht mehr hervorrufen. Bei der Besprechung der akuten Prostatitis folgt Zeissl der gewöhnlichen Einteilung in drei Stadien: katarrhalische, follikuläre und parenchymatöse Entzündung. Die chronische Prostatitis halt er ffir ausserordentlich häufig und geht soweit, zu behaupten, dass es keine chronische Gonorrhoe ohne Mitbeteiligung der Vorsteherdrüse gibt. Auch Erkrankungen der Samenblasen sind häufiger, als früher angenommen wurde. Sie sind meist einseitig und mit Prostatitis und Epididymitis gepaart. Ein diagnostisches Zeichen sind blutige Pollutionen mit himbeergeleeartigem Erguss. Bei der Therapie der Epididymitis und Orchitis gonorrh. warnt Verfasser vor dem Frickeschen Heftpflasterverband, durch den Shock-erscheiaungen beobachtet worden sind. Es folgt Besprechung des akuten und chronischen Blasenkatarrhs und der Komplíkationen von seiteri der Nieren. Bei den Strikturen unterscheidet er zwischen spastischen und organischen. Zur Behandlung der letzteren empfiehlt er die langsame Dilatation, ist diese nicht durchführbar, die Urethrotomia externa. Die Besprechung des wichtigen Gebiets der Harnröhrenverengerungen hätte im Verhältnis zu den iibrigen Kapiteln etwas ausführlicker sein können. Statt der Bezeiehnung spitzes Kondylom empfiehlt Zeissl den Namen venerisches Papillom, da mit Kondylom Dinge verschiedener Provenienz bezeichnet werden. Im Gegensatz zu anderen bezweifelt er übrigens dieÜbertragbarkeit des venerischenPapilloms. Gonorrhoe des Mastdarms ist beim Weibe häufiger als beim Manne, bei dem sie meistens durch $\Lambda$ vidernatürlichen Coitus in anum bedingt wird. Die von manche $\alpha$ beschriehene Tripperinfektion des Rachens und der Nase hat Verfasser niemals gesehen. Ausführlich wird auf die Frage des Ehekonsenses eingegangen. Derselbe ist nur dann zu verweigern, wenn durch mikroskopischen Befund oder Reinkultur noch Gonokokken gefunden werden. Trotz aller Behandlung noch zurückgebliebene leiehte katarrhalische Erscheinungen bilden keine Kontraindikationen. Von der Gonorrhoe zu unterscheiden ist die selten vor-komme'nde Urethritis non gonorrhoica. Dieselbe zeichnet sich aus durch schleichenden Verlauf und ist der Therapie wenig zugäuglich. Der zweite Teil des Buches ist dem Tripperprozess beim Weibe mit seinen zahlreichen Komplikationen gewidmet. Vulvitis, Vaginaltripper, die Gonorrhoe kleiner Mädchen, die Bartholinitis, der Tripper des Uterus, der Tuben, Ovarien, des Beckenbauchfells, die Peritonitis gonorrhoica und die Urethritis des Weibes werden ausführlich besprochen. Es folgt zum Schluss die Therapie des weiblichen Trippers. $\quad$ L. Lipman-Wulf-Berlin. 